\title{
Towards QCD running in 5 loops: quark mass anomalous dimension
}

\section{P. A. Baikov,}

Skobeltsyn Institute of Nuclear Physics, Lomonosov Moscow State University, 1(2),

Leninskie gory, Moscow 119991, Russian Federation

E-mail: baikovatheory.sinp.msu.ru

\section{K. G. Chetyrkin* and J. H. Kühn,}

Institut für Theoretische Teilchenphysik, Karlsruhe Institute of Technology (KIT), D-76128

Karlsruhe, Germany

E-mails : konstantin. chetyrkin@kit.edu

Johann.kuehn@kit.edu

We report first results of an ongoing project devoted to the analytical calculation of the QCD $\beta$-function and the quark mass anomalous dimension at the five loop level.

11th International Symposium on Radiative Corrections (Applications of Quantum Field Theory to Phenomenology)

22-27 September 2013

Lumley Castle Hotel, Durham, UK

\footnotetext{
* Speaker.
} 


\section{Introduction}

The method of the renormalization group ( $R G)[1,2,3]$ is of vital importance in modern quantum field theory. It is enough to recall that the famous idea of the asymptotic freedom is based on the RG concept of the running coupling constant. The RG functions $-\beta$-functions and various anomalous dimensions - serve as coefficients in the RG equations and are expressed in terms of Feynman Integrals (FI's). The complexity of these integrals grows drastically with the number of loops.

During last three decades or so there has been a tremendous progress in our ability to compute analytically the RG functions. The progress has been under way in, essentially, three directions.

i. General developments of our ability to deal with multiloop FI's. These have been thoroughly documented by Vladimir Smirnov in his bestseller books "Evaluating Feynman integrals" and "Feynman Integral Calculus" $[4,5]$ (see also [6, 7]). As a result two types of most relevant for RG calculations FI's, namely, massless propagators and (completely) massive tadpoles ( $\mathrm{p}$ - and m-integrals correspondingly) can be calculated (completely analytically) at the four loop level.

ii. Invention of special tools for significant simplifications of RG calculations. These include Infrared Rearrangement $[8,9,10]$ and $R^{*}$-operation [11].

iii. Continuous development of Computer Algebra Systems, with FORM [12] as most prominent and indispensable tool.

The current state of art of (analytical) RG calculations can be summarized as follows: generic four-loop RG calculations are now possible (see, e.g. [13, 14, 15, 16, 17, 18, 19]) and five-loop ones are gradually getting "feasible" [20,21].

In this talk we give the results of the first complete calculations of some of QCD RG functions at five loops. These are the quark mass and field anomalous dimensions and the anomalous dimension of the ghost field. The latter is one ingredient (among three) necessary for the construction of the five-loop contribution to the QCD $\beta$-function.

The precise evaluation of the quark mass anomalous dimension has important implications. The Higgs boson decay rate into charm and bottom quarks is proportional to the square of respective quark mass at the scale of $m_{H}$ and the uncertainty from the presently unknown 5-loop terms in the running of the quark mass is of order $10^{-3}$. This is comparable to the precision advocated for experiments e.g. at TLEP [22]. Similarly, the issue of Yukawa unification is affected by precise predictions for the anomalous quark mass dimension.

\section{Preliminaries}

Our starting point is the QCD Lagrangian with $n_{f}$ quark flavours written in terms of renormalized fields, coupling constant $g$ and quark massess $m_{f}$ :

$$
\begin{aligned}
\mathscr{L}_{0} & =-\frac{1}{4} Z_{3}\left(\partial_{\mu} A_{\nu}-\partial_{\nu} A_{\mu}\right)^{2}-\frac{1}{2} g Z_{1}^{3 g}\left(\partial_{\mu} A_{\nu}^{a}-\partial_{\nu} A_{\mu}^{a}\right)\left(A_{\mu} \times A_{v}\right)^{a} \\
& -\frac{1}{4} g^{2} Z_{1}^{4 g}\left(A_{\mu} \times A_{v}\right)^{2}-\frac{1}{2 \xi_{L}}\left(\partial_{\nu} A_{\mu}\right)^{2}+Z_{3}^{c} \partial_{\nu} \bar{c}\left(\partial_{\nu} c\right)+g Z_{1}^{c c g} \partial^{\mu} \bar{c}(A \times c) \\
& +Z_{2} \sum_{f=1}^{n_{f}} \bar{\psi}^{f} \mathrm{i} \not \partial \psi^{f}+g Z_{1}^{\psi \psi g} \sum_{f=1}^{n_{f}} \bar{\psi}^{f} A \psi^{f}-Z_{\psi \psi} \sum_{f=1}^{n_{f}} m_{f} \bar{\psi}^{f} \psi^{f},
\end{aligned}
$$


with bare gluon, quark and ghost fields related to the renormalized ones as follows:

$$
A_{0}^{a \mu}=\sqrt{Z_{3}} A^{a \mu}, \quad \psi_{0}^{f}=\sqrt{Z_{2}} \psi_{0}^{f}, \quad c_{0}^{a}=\sqrt{Z_{3}^{c}} c^{a} .
$$

The vertex Renormalization Constants (RCs)

$$
Z_{1}^{V}, \quad V \in\{3 \mathrm{~g}, 4 \mathrm{~g}, \mathrm{ccg}, \psi \psi \mathrm{g}\}
$$

are to be chosen to renormalize 3-gluon, 4-gluon, ghost-ghost-gluon, quark-quark-gluon vertex functions respectively. The Slavnov-Taylor identities allows one to express all vertex RCs in terms of wave function RCs and an independent charge RC, $Z_{g}=\frac{g_{0}}{g}$ :

$$
\begin{aligned}
Z_{\xi} & =Z_{3}, \\
Z_{g} & =\sqrt{Z_{1}^{4 g}}\left(Z_{3}\right)^{-1}, \\
Z_{g} & =Z_{1}^{3 g}\left(Z_{3}\right)^{-3 / 2}, \\
Z_{g} & =Z_{1}^{c c g}\left(Z_{3}\right)^{-1 / 2}\left(Z_{3}^{c}\right)^{-1}, \\
Z_{g} & =Z_{1}^{\psi \psi g}\left(Z_{3}\right)^{-1 / 2}\left(Z_{2}\right)^{-1} .
\end{aligned}
$$

Within the commonly accepted $\overline{\mathrm{MS}}$ scheme RCs are independent of dimensional parameters (masses and momenta) and can be represented as follows

$$
Z(h)=1+\sum_{i, j}^{1 \leq j \leq i} Z_{i j} \frac{h^{i}}{\mathcal{\varepsilon}^{j}},
$$

where $h=g^{2} /\left(16 \pi^{2}\right)$ and the parameter $\varepsilon$ is related to the continuous space time dimension $D$ via $D=4-2 \varepsilon$. Given a $\operatorname{RC} Z(h)$, the corresponding anomalous dimension is defined as

$$
\gamma(h)=-\mu^{2} \frac{\mathrm{d} \log Z(h)}{\mathrm{d} \mu^{2}}=\sum_{n=1}^{\infty} Z_{n, 1} n h^{n}=-\sum_{n=0}^{\infty}(\gamma)_{n} h^{n+1} .
$$

The anomalous dimension of the charge $h$ is conventionally referred to as "QCD $\beta$-function"; equations (2.8) imply that all four expressions in the Table below

$$
\begin{array}{|l|l|l|l|}
\hline \multicolumn{5}{|c|}{\beta(h)=} \\
\hline 2 \gamma_{1}^{c c g}-2 \gamma_{3}^{c}-\gamma_{3} & 2 \gamma_{1}^{\psi \psi g}-2 \gamma_{2}-\gamma_{3} & 2 \gamma_{1}^{3 g}-3 \gamma_{3} & \gamma_{1}^{4 g}-2 \gamma_{3} \\
\hline
\end{array}
$$

Table 1: Four different representation the QCD $\beta$-function.

can be used to find the QCD $\beta$-function $\beta(h)$. In real calculations only the first or the second possibility is usually employed.

To calculate the quark mass anomalous dimension, $\gamma_{m}$, one needs to find the so-called quark mass renormalization constant, $Z_{m}$, which is defined as the ratio of the bare and renormalized quark masses, viz.

$$
Z_{m}=\frac{m_{f}^{0}}{m_{f}}=\frac{Z_{\psi \psi}}{Z_{2}} .
$$

The final formula for $\gamma_{m}$ reads

$$
\gamma_{m}=\gamma_{\psi \psi}-\gamma_{2}
$$




\section{Five-loop running of the ghost field}

As a first step towards five-loop QCD $\beta$-function we have computed the anomalous dimension of the ghost field

$$
\gamma_{3}^{c}=-\sum_{i=0}^{\infty}\left(\gamma_{3}^{c}\right)_{i} h^{i+1}
$$

The anomalous dimension is known up to four loops from the works [23, 24]. The new five-loop coefficient reads (in the Feynman gauge):

$$
\begin{aligned}
\left(\gamma_{3}^{c}\right)_{4} & =-\frac{193301287}{2048}-\frac{19562145}{128} \zeta_{3}-\frac{2060829}{128} \zeta_{3}^{2}+\frac{1101573}{16} \zeta_{4} \\
& +\frac{66632427}{128} \zeta_{5}-\frac{36327825}{256} \zeta_{6}-\frac{140900823}{512} \zeta_{7} \\
& +n_{f}\left[\frac{633704171}{27648}+\frac{5166473}{144} \zeta_{3}+\frac{233519}{64} \zeta_{3}^{2}-\frac{764949}{32} \zeta_{4}\right. \\
& \left.-\frac{32902291}{384} \zeta_{5}+\frac{4123825}{128} \zeta_{6}+\frac{14425075}{384} \zeta_{7}\right] \\
& +n_{f}^{2}\left[-\frac{1326547}{3456}-\frac{1739167}{864} \zeta_{3}-\frac{2659}{6} \zeta_{3}^{2}+\frac{13485}{8} \zeta_{4}+\frac{8074}{9} \zeta_{5}-\frac{16775}{12} \zeta_{6}\right] \\
& +n_{f}^{3}\left[-\frac{342895}{7776}-\frac{1211}{18} \zeta_{3}-\frac{5}{2} \zeta_{4}+\frac{284}{3} \zeta_{5}\right]+n_{f}^{4}\left[\frac{65}{108}+\frac{20}{27} \zeta_{3}-\frac{4}{3} \zeta_{4}\right]
\end{aligned}
$$

Numerically $\left(a_{s} \equiv \frac{\alpha_{s}}{\pi} \equiv 4 h\right)$ :

$$
\gamma_{3}^{c}\left(n_{f}=3\right)=\frac{3}{8}\left(a_{s}+2.4375 a_{s}^{2}+4.8867 a_{s}^{3}+19.980 a_{s}^{4}+122.246 a_{s}^{5}\right) .
$$

For generic $n_{f}$ :

$$
\begin{aligned}
& \gamma_{3}^{c}=\frac{3}{8}\left\{a_{s}+a_{s}^{2}\left(3.063-0.208 n_{f}\right)+a_{s}^{3}\left(10.556-1.768 n_{f}-0.0405 n_{f}^{2}\right)\right. \\
+ & a_{s}^{4}\left(49.325-10.957 n_{f}+0.36562 n_{f}^{2}+0.0087 n_{f}^{3}\right) \\
& \left.+a_{s}^{5}\left(283.632-70.979 n_{f}+5.498 n_{f}^{2}+0.0769 n_{f}^{3}-0.000128038 n_{f}^{4}\right)\right\}
\end{aligned}
$$

It is instructive to observe that significant cancellations between $n_{f}^{0}$ and $n_{f}^{1}$ terms for the values of $n_{f}$ around 3 or so persist also at five-loop order.

\section{Five-loop quark mass anomalous dimension}

The quark mass anomalous dimension is known to four loops from the works [25, 26]. Our result for the five-loop coefficient in

$$
\gamma_{m}=-\sum_{i=0}^{\infty}\left(\gamma_{m}\right)_{i} h^{i+1}
$$


reads (note that $\gamma^{m}$ is a gauge independent quantity):

$$
\begin{aligned}
\left(\gamma_{m}\right)_{4}= & \frac{99512327}{162}+\frac{46402466}{243} \zeta_{3}+96800 \zeta_{3}^{2}-\frac{698126}{9} \zeta_{4} \\
& -\frac{231757160}{243} \zeta_{5}+242000 \zeta_{6}+412720 \zeta_{7} \\
+ & n_{f}\left[-\frac{150736283}{1458}-\frac{12538016}{81} \zeta_{3}-\frac{75680}{9} \zeta_{3}^{2}+\frac{2038742}{27} \zeta_{4}\right. \\
& \left.\quad+\frac{49876180}{243} \zeta_{5}-\frac{638000}{9} \zeta_{6}-\frac{1820000}{27} \zeta_{7}\right] \\
+ & n_{f}^{2}\left[\frac{1320742}{729}+\frac{2010824}{243} \zeta_{3}+\frac{46400}{27} \zeta_{3}^{2}-\frac{166300}{27} \zeta_{4}-\frac{264040}{81} \zeta_{5}+\frac{92000}{27} \zeta_{6}\right] \\
+ & n_{f}^{3}\left[\frac{91865}{1458}+\frac{12848}{81} \zeta_{3}+\frac{448}{9} \zeta_{4}-\frac{5120}{27} \zeta_{5}\right]+n_{f}^{4}\left[-\frac{260}{243}-\frac{320}{243} \zeta_{3}+\frac{64}{27} \zeta_{4}\right] .
\end{aligned}
$$

Note that the boxed terms are in full agreement with predictions made on the basis of the $1 / n_{f}$ method in $[27,28]$.

In numerical form $\gamma_{m}$ reads

$$
\begin{aligned}
\gamma_{m}= & -a_{s}-a_{s}^{2}\left(4.20833-0.138889 n_{f}\right) \\
& -a_{s}^{3}\left(19.5156-2.28412 n_{f}-0.0270062 n_{f}^{2}\right) \\
& -a_{s}^{4}\left(98.9434-19.1075 n_{f}+0.276163 n_{f}^{2}+0.00579322 n_{f}^{3}\right) \\
& -559.71+143.6 n_{f}-7.4824 n_{f}^{2}-0.1083 n_{f}^{3}+0.00008535 n_{f}^{4}
\end{aligned}
$$

and

$$
\begin{aligned}
& \gamma_{m} \overline{\overline{n_{f}=3}}-a_{s}-3.79167 a_{s}^{2}-12.4202 a_{s}^{3}-44.2629 a_{s}^{4}-198.907 a_{s}^{5}, \\
& g_{m} \overline{\overline{n_{f}=4}}-a_{s}-3.65278 a_{s}^{2}-9.94704 a_{s}^{3}-27.3029 a_{s}^{4}-111.59 a_{s}^{5}, \\
& g_{m} \overline{\overline{n_{f}=5}}-a_{s}-3.51389 a_{s}^{2}-7.41986 a_{s}^{3}-11.0343 a_{s}^{4}-41.8205 a_{s}^{5}, \\
& \gamma_{m} \overline{\overline{n_{f}=6}}-a_{s}-3.375 a_{s}^{2}-4.83867 a_{s}^{3}+4.50817 a_{s}^{4}+9.76016 a_{s}^{5} .
\end{aligned}
$$

Inspection of eqs. (4.3) shows quite moderate growth of the series in $a_{s}$ appearing in the quark mass anomalous dimension at various values of active quark flavours (recall that even for scales as small as $2 \mathrm{GeV} a_{s} \equiv \frac{\alpha_{s}}{\pi} \approx 0.1$ ).

Finally, our result for the anomalous dimension of the quark field $\gamma_{2}=-\sum_{i=0}^{\infty}\left(\gamma_{2}\right)_{i} h^{i+1}$ reads 
(again in the Feynman gauge):

$$
\begin{aligned}
&\left(\gamma_{2}\right)_{4}=\frac{2798900231}{7776}+\frac{17969627}{864} \zeta_{3}+\frac{13214911}{648} \zeta_{3}^{2}+\frac{16730765}{864} \zeta_{4}-\frac{832567417}{3888} \zeta_{5} \\
& \quad+\frac{40109575}{1296} \zeta_{6}+\frac{124597529}{1728} \zeta_{7} \\
&+n_{f}\left[-\frac{861347053}{11664}-\frac{274621439}{11664} \zeta_{3}+\frac{1960337}{972} \zeta_{3}^{2}+\frac{465395}{1296} \zeta_{4}\right. \\
&\left.\quad+\frac{22169149}{5832} \zeta_{5}+\frac{1278475}{1944} \zeta_{6}+\frac{3443909}{216} \zeta_{7}\right] \\
&+n_{f}^{2}\left[\frac{37300355}{11664}+\frac{1349831}{486} \zeta_{3}-\frac{128}{9} \zeta_{3}^{2}-\frac{27415}{54} \zeta_{4}-\frac{12079}{27} \zeta_{5}-\frac{800}{9} \zeta_{6}-\frac{1323}{2} \zeta_{7}\right] \\
&+ n_{f}^{3}\left[-\frac{114049}{8748}-\frac{1396}{81} \zeta_{3}+\frac{208}{9} \zeta_{4}\right]+n_{f}^{4}\left[\frac{332}{729}-\frac{64}{81} \zeta_{3}\right]
\end{aligned}
$$

The coefficients $\left(\gamma_{2}\right)_{i}$ with $i \leq 3$ can be found in [29] (for the case of a general covariant gauge and $\mathrm{SU}(\mathrm{N})$ gauge group).

\section{Technical tools}

As is well-known evaluation of any $L$-loop anomalous dimension in the $\overline{\mathrm{MS}}$-scheme can be reduced, with the help of the $R^{*}$-operation [11], to the evaluation of some $L-1$-loop massless propagators [30]. In our case $\mathrm{L}=5$ and we need to be able effectively compute a host of four-loop massless propagators (that is p-integrals). These, in turn, can be reduced to 28 master integrals. The reduction is based on evaluating sufficiently many terms of the $1 / D$ expansion [31] of the corresponding coefficient functions [32]. The master integrals are known analytically from [33, 34].

Note that all our calculations have been performed on a SGI ALTIX 24-node IB-interconnected cluster of 8-cores Xeon computers using parallel MPI-based [35] as well as thread-based [36] versions of FORM [12].

\section{Conclusions}

Unfortunately, at the moment it is not possible to take self-consistently into account our fiveloop result for $\gamma_{m}$ for the quark mass running: this requires the knowledge of the five-loop QCD $\beta$-function. The latter problem is under calculation in our group.

K.G.C. thanks J. Gracey and members of the DESY-Zeuthen theory seminar for usefull discussions.

This work was supported by the Deutsche Forschungsgemeinschaft in the Sonderforschungsbereich/Transregio SFB/TR-9 “Computational Particle Physics”, by RFBR (grant 11-02-01196).

\section{References}

[1] E. Stueckelberg and A. Petermann, La normalisation des constantes dans la theorie des quanta, Helv. Phys. Acta. 26 (1953) 499-520. 
[2] M. Gell-Mann and F. Low, Quantum electrodynamics at small distances, Phys.Rev. 95 (1954) $1300-1312$.

[3] N. Bogolyubov and D. Shirkov, Charge renormalization group in quantum field theory, Nuovo Cim. 3 (1956) 845-863.

[4] V. A. Smirnov, Evaluating Feynman integrals. Springer, Berlin, 2004.

[5] V. A. Smirnov, Feynman Integral Calculus. Springer, Berlin, 2006.

[6] V. A. Smirnov, Applied asymptotic expansions in momenta and masses. Springer, Berlin, 2002.

[7] V. A. Smirnov, Analytic tools for Feynman integrals. Springer, Berlin, 2012.

[8] A. A. Vladimirov, Method For Computing Renormalization Group Functions In Dimensional Renormalization Scheme, Theor. Math. Phys. 43 (1980) 417.

[9] K. G. Chetyrkin, A. L. Kataev, and F. V. Tkachov, New Approach to Evaluation of Multiloop Feynman Integrals: The Gegenbauer Polynomial x Space Technique, Nucl. Phys. B174 (1980) 345-377.

[10] O. Tarasov and A. Vladimirov, Three Loop Calculations in Non-Abelian Gauge Theories, Phys.Part.Nucl. 44 (2013) 791-802, [arXiv:1301.5645].

[11] K. G. Chetyrkin and V. A. Smirnov, R* OPERATION CORRECTED, Phys. Lett. B144 (1984) 419-424.

[12] J. A. M. Vermaseren, New features of form, math-ph/0010025.

[13] S. G. Gorishny, A. L. Kataev, S. A. Larin, and L. R. Surguladze, The analytical four loop corrections to the qed beta function in the ms scheme and to the qed psi function: Total reevaluation, Phys. Lett. B256 (1991) 81-86.

[14] K. G. Chetyrkin, Correlator of the quark scalar currents and gamma(tot)(h-> hadrons) at o(alpha(s)**3) in pqcd, Phys. Lett. B390 (1997) 309-317, [hep-ph/9608318].

[15] T. van Ritbergen, J. A. M. Vermaseren, and S. A. Larin, The four-loop beta function in quantum chromodynamics, Phys. Lett. B400 (1997) 379-384, [hep-ph/9701390].

[16] Y. Schroder, Automatic reduction of four-loop bubbles, Nucl. Phys. Proc. Suppl. 116 (2003) 402-406, [hep-ph/0211288].

[17] M. Czakon, U. Haisch, and M. Misiak, Four-Loop Anomalous Dimensions for Radiative Flavour-Changing Decays, JHEP 0703 (2007) 008, [hep-ph / 061232 ]].

[18] V. Velizhanin, Four loop anomalous dimension of the second moment of the non-singlet twist-2 operator in QCD, Nucl.Phys. B860 (2012) 288-294, [arXiv: 1112.3954 ].

[19] Z. Bern, S. Davies, T. Dennen, A. V. Smirnov, and V. A. Smirnov, The Ultraviolet Properties of $N=4$ Supergravity at Four Loops, arXiv:1309.2498.

[20] P. Baikov, K. Chetyrkin, J. Kuhn, and J. Rittinger, Vector Correlator in Massless QCD at Order $\mathscr{O}\left(\alpha_{s}^{4}\right)$ and the QED beta-function at Five Loop, JHEP 1207 (2012) 017, [arXiv: 1206.1284 ].

[21] B. Eden, P. Heslop, G. P. Korchemsky, V. A. Smirnov, and E. Sokatchev, Five-loop Konishi in N=4 SYM, Nucl.Phys. B862 (2012) 123-166, [arXiv: 1202 . 5733].

[22] M. Bicer, H. Duran Yildiz, I. Yildiz, G. Coignet, M. Delmastro, et al., First Look at the Physics Case of TLEP, arXiv:1308.6176. 
[23] K. G. Chetyrkin, Four-loop renormalization of QCD: Full set of renormalization constants and anomalous dimensions, Nucl. Phys. B710 (2005) 499-510, [hep-ph/ 0405193$].$

[24] M. Czakon, The four-loop QCD beta-function and anomalous dimensions, Nucl. Phys. B710 (2005) 485-498, [hep-ph/0411261].

[25] K. G. Chetyrkin, Quark mass anomalous dimension to O(alpha(s)**4), Phys. Lett. B404 (1997) 161-165, [hep-ph/9703278].

[26] J. A. M. Vermaseren, S. A. Larin, and T. van Ritbergen, The 4-loop quark mass anomalous dimension and the invariant quark mass, Phys. Lett. B405 (1997) 327-333, [hep-ph/9703284].

[27] M. Ciuchini, S. E. Derkachov, J. Gracey, and A. Manashov, Computation of quark mass anomalous dimension at $O\left(1 / N^{* * 2(f))}\right.$ in quantum chromodynamics, Nucl.Phys. $\mathbf{B 5 7 9}$ (2000) 56-100, [hep-ph/9912221].

[28] M. Ciuchini, S. E. Derkachov, J. Gracey, and A. Manashov, Quark mass anomalous dimension at $O(1 / N(f) * * 2)$ in QCD, Phys.Lett. B458 (1999) 117-126, [hep-ph/9903410].

[29] K. G. Chetyrkin and A. Retey, Renormalization and running of quark mass and field in the regularization invariant and MS-bar schemes at three and four loops, Nucl. Phys. B583 (2000) 3-34, [hep-ph/9910332].

[30] K. G. Chetyrkin, Corrections of order alpha(s)**3 to R(had) in pQCD with light gluinos, Phys. Lett. B391 (1997) 402-412, [hep-ph/9608480].

[31] P. A. Baikov, A practical criterion of irreducibility of multi-loop feynman integrals, Phys. Lett. B634 (2006) 325-329, [hep-ph/0507053].

[32] P. A. Baikov, Explicit solutions of the 3-loop vacuum integral recurrence relations, Phys. Lett. $\mathbf{B 3 8 5}$ (1996) 404-410, [hep-ph/9603267].

[33] P. A. Baikov and K. G. Chetyrkin, Four Loop Massless Propagators: an Algebraic Evaluation of All Master Integrals, Nucl. Phys. B837 (2010) 186-220, [arXiv: 1004 .1153].

[34] R. N. Lee, A. V. Smirnov, and V. A. Smirnov, Master Integrals for Four-Loop Massless Propagators up to Transcendentality Weight Twelve, Nucl. Phys. B856 (2012) 95-110, [arXiv: 1108.0732 ].

[35] M. Tentyukov et al., ParFORM: Parallel Version of the Symbolic Manipulation Program FORM, Cs/ 0407066 .

[36] M. Tentyukov and J. A. M. Vermaseren, The multithreaded version of FORM, hep-ph/0702279. 